Check for updates

Cite this: RSC Adv., 2017, 7, 18668

Received 9th January 2017

Accepted 13th March 2017

DOI: $10.1039 / \mathrm{c} 7 \mathrm{ra00336f}$

rsc.li/rsc-advances

\section{Stem cell therapies for ischemic stroke: current animal models, clinical trials and biomaterials}

\author{
Hugh H. Chan, ${ }^{\text {ab }}$ Connor A. Wathen, ${ }^{c}$ Ming Ni ${ }^{\star a}$ and Shuangmu Zhuo (D)*a
}

Ischemic stroke is one of the most common causes of death. A brain that has suffered a stroke is significantly damaged due to the transient interruption of blood supply. Stroke survivors suffer from stroke-induced cognitive, motor and psychiatric problems that require increased use of the health care system, which results in a very poor quality of life and a heavy emotional and financial burden for patients and their families. Although rehabilitation can ameliorate some behavioral abnormalities, it does not significantly improve stroke symptoms. Neuroregeneration may be the principle process for replenishing of lost brain functions. Stem cell therapy is a novel approach to restore neurological function following ischemic stroke. Our goal in this review is to first briefly introduce the pathophysiology of stroke then document the progress of stem cell research, including clinical trials, towards the treatment of ischemic stroke. We highlight the promising applications of biomaterials to enhance the therapeutic effect of stem cell therapy in stroke treatment. As biomaterials provide efficient scaffolds for the growth and differentiation of stem cells, they may guarantee the quality and yield of stem cells for grafting. Furthermore, biomaterials also provide a convenient method for delivering the stem cells to be transplanted. This review provides direction for future research to combine tissue engineering approaches with stem cell therapy for the treatment of stroke.

\section{Introduction}

Stroke is one of the most common causes of death in developed countries, ${ }^{1}$ and occurs primarily in the elderly population with a higher risk in males. ${ }^{2}$ Ischemic stroke occurs when any of the many arteries perfusing the brain is occluded, resulting in anoxic damage to the portion of the brain supplied by the occluded artery. ${ }^{3}$ Even if there is reperfusion of the ischemic tissue, the presence of highly oxygenated blood allows for the production of reactive oxygen species which results in oxidative damage. ${ }^{4}$ The combination of ischemic and oxidative damage leads to neuronal death, including apoptosis, ${ }^{5}$ and loss of neural function. Because the location of the stroke is determined by the vessel that is occluded and frequently spans
${ }^{a}$ Key Laboratory of OptoElectronic Science and Technology for Medicine of Ministry of Education \& Fujian Provincial Key Laboratory of Photonics Technology, Fujian Normal University, Fuzhou 350007, P. R. China. E-mail: shuangmuzhuo@gmail. com; mingni.sg@gmail.com
${ }^{b}$ Department of Neuroscience, Lerner Research Institute, Cleveland Clinic, Cleveland, Ohio, USA

${ }^{c}$ Center for Neurological Restoration, Cleveland Clinic, Cleveland, Ohio, USA

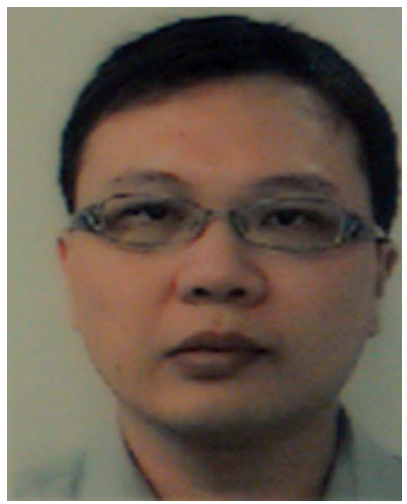

Hugh $H$ Chan received his $P h D$ from Imperial College London in 2010. He is currently a postdoctoral fellow at the Neurosciences Department of the Lerner Research Institute of Cleveland Clinic. His current research focuses on rehabilitation and repair in experimental stroke models.

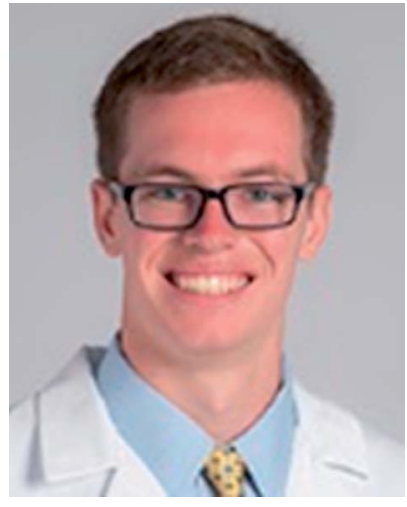

Connor A Wathen received his $B S$ from the University of Notre Dame in 2013. He is currently a medical student at the Cleveland Clinic Lerner College of Medicine of the Case Western Reserve University and will receive his $M D$ in 2018. His current research focuses on deep cerebellar stimulation to improve post-stroke rehabilitation. 
several functional domains, the resulting symptoms are usually a combination of motor, cognitive and psychiatric deficits. ${ }^{6-11}$

Although there are emerging treatments that restore perfusion to the ischemic brain, including the administration of tissue plasminogen activator to dissolve thrombi and emboli as well as intra-arterial/endovascular procedures to re-canalize blood vessels, ${ }^{12,13}$ these types of treatment primarily serve to reduce stroke mortality. As neurological injury due to ischemia is largely irreversible, it is necessary to develop novel therapeutic approaches to restore lost neurological functions through the regeneration of neurons. ${ }^{14} \mathrm{~A}$ few studies that have provided evidence on the therapeutic effect of transplantation of different types of stem cells have been documented, in which the stroke symptoms were relieved and the lesion volumes were reduced. ${ }^{15,16}$ These studies suggested that the transplantation of stem cells could be a promising approach for the treatment of stroke. Currently, however, there are still problems associated with stem cell therapies for stroke. In this review, we will highlight the obstacles encountered during the development of such therapies. Transplanted stem cells often struggle to survive after injection, and biomaterials provide a perfect solution as scaffolds/delivery vehicles for cells to safely arrive at the injured sites. We suggest and discuss the applications of biomaterials to stem cell therapy in the treatment of stroke, which may provide a better understanding of the direction of research in the field of stem cell therapy for stroke.

\section{Stem cell research in animal models}

Although there is a small degree of spontaneous cellular regeneration following ischemic stroke, the amount of regeneration is not sufficient for functional neurological restoration. The administration of exogenous stem cells has the potential to overcome this limitation and provide neurological restoration in the compromised brain following stroke. The transplantation of stem cells from different sources has been evaluated in order to determine their efficacy and safety in the treatment of stroke. The goal of therapy is to develop stem cell therapy for (1) restoring compromised behavioral deficits and (2) restructuring the damaged brain regions. There are five primary stem cell types that are the focus of the development of stem cell therapy of stroke, namely embryonic stem cells (ESCs), neural stem cells (NSCs), mesenchymal stem cells (MSCs), hematopoietic stem cells (HSCs) and induced pluripotent stem cells (iPSCs). Table 1 summarizes the usage of different types of stem cells in stem cell therapy research for ischemic stroke.

\subsection{Endogenous stem cells}

Although stroke induces brain tissue death, as compensation, there is a small degree of new cell formation, particularly neurons, after stroke. ${ }^{17}$ These endogenous stem cells, however, are not sufficient for recovery. In an experimental stroke model in rodents, young rats were shown to exhibit a higher level of new neuron formation, termed neurogenesis, than that in aged rats. ${ }^{18}$ To support neurogenesis after stroke, new blood vessels also form in order to reconstruct the compromised circulation, a process known as angiogenesis. ${ }^{19,20}$ Neurogenesis and angiogenesis are more prevalent in young brains, particularly at the postnatal to adolescent stages. Although most strokes occur in older individuals, limited degrees of neurogenesis and angiogenesis are still detected in adult brains. ${ }^{21,22}$ From the studies of rodents, the neurogenesis triggered by stroke occurs in the subventricular zone (SVZ) and subgranular zone (SGZ). ${ }^{23,24}$ Stem cells or neural progenitor cells (NPCs) divide and differentiate in the SVZ adjacent to the lateral ventricles..$^{25}$ Guided by chemokines such as vascular endothelial growth factors, stem cell factor, and monocyte chemoattractant protein 1 , NPCs migrate to the penumbra area that surrounds the ischemic lesion. ${ }^{26}$ NPCs are characterized by certain markers such as doublecortin, nestin, Sox 2 , and others. ${ }^{27,28}$ NPCs derived from the SGZ divide and develop into mature neurons then migrate to the hippocampus and other parts of the limbic system..$^{29}$ Angiogenesis is primarily characterized by the formation of endothelial cells. New blood vessels are formed from pre-existing blood vessels, and elongate to access the sites at which neurogenesis occurs. ${ }^{30}$ De novo blood vessels can also form without the aid of pre-existing blood vessels, a process known as vasculogenesis. ${ }^{31}$

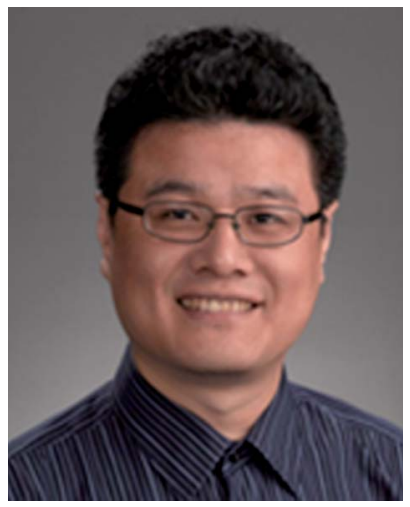

Ming Ni received his Ph.D. in Chemical Engineering from the University of Washington in 2004. He is currently a research scientist at the Institute of Bioengineering and Nanotechnology, Agency for Science, Technology and Research in Singapore. His current research focuses on biomaterials and nanomaterials and their applications as probes for nonlinear optical microscopy and super high resolution microscopy. He is also interested in stem cell therapy and drug delivery.

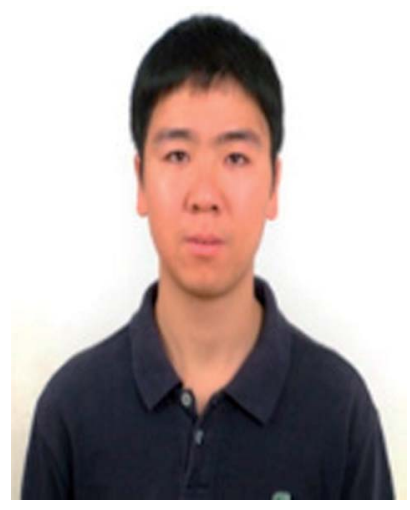

Shuangmu Zhuo received his Ph.D. degree in Optics Engineering from the Fujian Normal University, China, in 2012. He then joined the Singapore-MIT Alliance for Research and Technology as a Postdoctoral Research Fellow. He is currently a Professor at the College of Photonic and Electronic Engineering, Fujian Normal University, China. His research interests include the development and applications of nonlinear optical microscopy in biological and biomedical research. 
Table 1 Comparison of the different types of stem cells developed to date, and their likelihood to induce immune reaction and tumorigenesis, proliferative ability, differentiability and general efficacy for stroke therapy

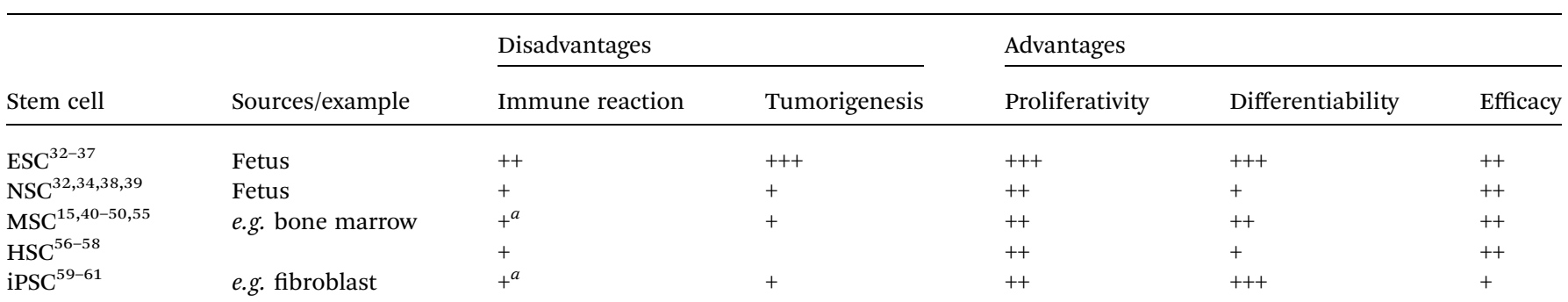

${ }^{a}$ When the MSCs and iPSCs are derived from the recipients' somatic cells, there is hypothetically no immune response.

\subsection{Exogenous stem cells}

2.2.1 Embryonic stem cells (ESCs). ESCs, harvested from the inner cell mass of embryos, demonstrate the capability for unlimited self-renewal and a strong potential for differentiation. Hypothetically, ESCs can develop into any cell types depending on the conditions of development. Under specific conditions, ESCs can differentiate into neural lineage. ${ }^{32-34}$ For instance, the administration of fibroblast growth factor 2 , but not epidermal growth factor and leukemia inhibitory factor, induces the differentiation of ESCs to neuronal cell types, which express glutamatergic, GABAergic or dopaminergic markers. ${ }^{34}$ Thus, ESCs are considered to be a promising source of stem cells for transplantation into the degenerating brain. One early study showed that intrastriatal grafting of mouse ESCs, conditioned to differentiate into neuronal like cells, rescued the ischemic lesion and partially restored motor function in a middle cerebral artery occlusion (MCAO) rat model of ischemic stroke. ${ }^{35}$ Another approach is to apply ESCs directly to the cerebral cortex, which is usually severely damaged from stroke lesion. The application of this technique successfully improved the motor and sensory function of rats following MCAO stroke induction. ${ }^{36}$ Due to the remarkable ability for selfrenewal and differentiation, however, malignant transformation or tumorigenesis induced by ESCs prevents the grafting of ESCs that are used to treat of stroke. ${ }^{37}$

2.2.2 Neural stem cells (NSCs). NSCs harvested from postnatal animals may provide a less proliferative but more neural specific stem cell for transplantation. Unfortunately, the yield of NSCs obtained from an individual rodent is quite small as the cells are only found in the SVZ and SGZ. To overcome this obstacle, induced differentiation of ESCs can provide a higher yield of NSCs. Partially differentiated ESCs, namely ESC-derived NSCs, were developed to reduce the risk of tumorigenesis as observed in the transplantation of ESCs as discussed earlier. ${ }^{38}$ Neural specific conditions are first applied to allow the ESCs to differentiate into neural related cell types prior to transplantation. $^{32-34}$ The subsequent transplantation of ESC-derived NSCs into the rat cortex improved MCAO-induced asymmetric limb usages, improved the latency on the rotarod task for motor control, and even reduced the volume of the stroke lesion. ${ }^{39}$

2.2.3 Mesenchymal stem cells (MSCs). MSCs are not as limited, in terms of their potential for differentiation and selfrenewal, as NSCs. Their wider availability also makes MSCs an attractive option for stem cell therapy of stroke. MSCs are stem cells, typically derived from bone marrow, placenta, heart and adipose tissue, skeletal muscle, and pancreas tissue, all of which are broadly distributed in the body thus making them easier to harvest. ${ }^{\mathbf{4 0 - 4 3}}$ Although MSCs originate from bone marrow, they have the potential to differentiate into neural cell types. ${ }^{44}$ A number of studies concerning the application of MSCs in rodent models of stroke have been published, which document the improvement of motor and cognitive symptoms. ${ }^{15,45}$ Instead of differentiating into neural cells to restore the motor deficits in the stroke models, ${ }^{\mathbf{4 6}}$ transplantation of MSCs could induce multiple mechanisms of stroke recovery. For instance, transplantation of MSCs into MCAO stroked rats also produces motor recovery via an increase of angiogenesis. ${ }^{47}$ Interestingly, MSC therapy can also reduce stroke-induced apoptosis. ${ }^{48}$ In addition to direct differentiation into neural cell types, MSCs mediate the release of neurotrophic factors, such as brain derived neurotrophic factor (BDNF), which further promote stroke recovery. ${ }^{\mathbf{4 6}}$ Additionally, genetically modified MSCs which overexpress BDNF promise better therapeutic effects than unmodified MSCs.

The therapeutic effect of bone marrow mononuclear cells (BMMNCs) in stroke has also been frequently studied in rodent models of ischemic stroke. In a rat MCAO model, transplantation of BMMNCs at the early stages of stroke reduced infarct volume, oxidative damage, and neuroinflammation, and the effects were enhanced by valproic acid. ${ }^{49}$ Furthermore, 5fluorouracil pre-treated BMMNCs were also effective in reducing stroke lesion volume. ${ }^{50}$ Recently, MSCs from human dental pulp stem cells were found to have a more potent effect on the protection of ischemic human astrocyte and in the rodent stroke model, ${ }^{51,52}$ thus indicating that it could be a better source of MSCs. ${ }^{53}$

Current research now focuses on alternative administration routes of MSCs. Instead of focal administration of MSCs into stroked brains, systemic administration of MSCs may provide a less invasive approach for the delivery of stem cells. The safety of intra-arterial infusion of human bone marrow-derived MSCs into the external carotid artery in the MCAO rat brain model has been documented. ${ }^{54}$ Although there was only a transient peak distribution of transplanted MSCs in the brain, a significantly higher amount of MSCs was documented in the ipsilesional cerebral cortex. Another remarkable study investigating 
intravenous administration of allogenic MSCs derived from bone marrow and adipose tissue also demonstrated functional recovery following stroke. ${ }^{55}$ Furthermore, MSC transplants were also associated with a reduction in apoptosis and increase in cellular plasticity. These findings strongly suggested that peripheral administration of MSCs is possibly a safe and effective approach for stem cell transplantation.

2.2.4 Hematopoietic stem cells (HSCs). These are another type of MSCs derived from bone marrow, which differentiate into different red blood cells and lymphoid cells. ${ }^{56}$ HSCs are also one of the cell populations found in BMMNCs. In an MCAO mouse model, HSCs reduced the ischemic infarct volume at the cerebral cortex. ${ }^{57}$ Under the stimulation of granulocyte-colony stimulating factor and stem cell factor, HSCs reduced atrophy in the ipsilesional cerebral hemisphere in a hypoxia-ischemia model. ${ }^{\mathbf{5 8}}$ These findings suggest that HSCs are also potentially beneficial for ameliorating ischemic stroke induced degeneration.

2.2.5 iPSCs. Another source which guarantees a high yield of stem cells is to reprogram somatic cells to become iPSCs. By using four crucial factors, namely Oct3/4, Sox2, c-Myc and Klf4, somatic cells can be induced to exhibit the morphology of ESCs and express related markers. ${ }^{59}$ It has been shown that intradural transplantation of iPSCs into the brain of an MCAO rat improves motor performance for the rotarod task. ${ }^{60}$ Furthermore, human fibroblast-derived iPSCs grafted to rats following MCAO stroke injury managed to migrate to the lesioned area and partially restore sensorimotor function. ${ }^{61}$ Although the use of iPSCs in stroke treatment is relatively new, their properties of rapid proliferation and multipotential differentiation indicate that iPSCs are very promising for stroke treatment.

\section{Clinical application of stem cells towards stroke}

\subsection{Current trials}

The clinical safety, including tumorigenesis and the immune response, of stem cells therapies for stroke has been reported in a series of studies. ${ }^{\mathbf{6 2}, 63}$ In one clinical study, autologous MSCs were intravenously delivered months after stroke. ${ }^{64}$ In this study, the lesion volume detected by MRI was reduced by $20 \%$. Remarkably, there was no tumorigenesis, abnormal cell growth or venous embolism observed. The safety of this study and another similar pilot clinical trial demonstrated the encouraging potential of the application of MSCs in clinical settings. ${ }^{65}$ In another clinical study on stem cell therapy for acute stroke, mononuclear cells derived from bone marrow improved the clinical outcomes as shown by a reduction in the National Institute of Health Stroke Scale (NIHSS) score 6 months after grafting. ${ }^{66}$

As mentioned previously, there are numerous studies on the efficacy of bone marrow-derived mononuclear cells in rodent models of stroke. ${ }^{\mathbf{4 9}, 50}$ Promising results have led to clinical trials of the BMMNCs. For instance, in a clinical study of autologous BMMNCs, the transplanted BMMNCs improved the prognosis of functional recovery in patients with chronic stroke (Table 2). ${ }^{67}$
The intravenous transplantation of autologous BMMNCs was also proven to be clinically safe in a pilot clinical trial; ${ }^{68}$ unfortunately there was no significant therapeutic effect in the patients (Table 2). ${ }^{69}$ In a later stage clinical study, autologous BMMNCs improved the cerebral blood flow and neurological recovery (Table 2). ${ }^{70}$ Although the restorative effect of BMMNCs only shows a small degree of therapeutic effect and remains controversial, clinical trials involving MSCs are the focus of development of stem cell therapies for stroke. ${ }^{62,63,65-67,69-71}$ Specifically, two meta-analyses of the efficacy of transplantation of autologous BMMNCs (one pre-clinical and another based on single arm proportional meta-analysis) in stroke patients have been reviewed. ${ }^{72,73}$

Currently, ReNeuron, a British based company, is sponsoring a phase I clinical trial investigating the clinical usage of a novel NSC, CTX0E03, in stroke treatment, and it has recently been announced that the study has been completed. Although the company has not disclosed any results, the phase II stage of the study of CTX0E03 has been registered. Table 2 contains a summary of the recent clinical trials of stem cell therapies for stroke.

\subsection{Obstacles}

3.2.1 Immune response. Transplantation of any foreign cells, including stem cells, leads to an immune reaction. This significantly reduces the survival rate of transplanted stem cells, which are removed by the immune system. Although immunosuppressants can partially eliminate this unwanted effect, transplant cell loss cannot be avoided if transplanting ESCs, NSCs or MSCs from another individual donor. However, the administration of iPSCs derived from the somatic cells of the transplant recipient, HSCs or MSCs could overcome this immune reaction. ${ }^{71}$ Somatic cells, such as fibroblasts, can first be obtained from the patient and subsequently reprogrammed to function as optimal stem cells such as NSCs, using molecular interference approaches. As mentioned earlier, the reprogramming of stem cells can be induced by the transfection of Oct3/4, Sox2, c-Myc and Klf4 factors. ${ }^{59}$ Additional molecular techniques, such as the introduction of genes for the expression of neurotrophic factors, can also be applied. iPSCs can thus provide immune reaction-free and specifically tailored stem cell therapy. ${ }^{45}$ As mentioned above, MSCs can be obtained from various peripheral tissues of an individual. ${ }^{\mathbf{4 1}}$ Thus, autologous MSCs are another potential source of stem cells that produce a less severe immune reaction following transplantation. ${ }^{64}$ Interestingly, some studies showed that there were no such immune problems in the transplantation of allogenic stem cells. For instance, allogenic mesenchymal stem cells did not induce any immune rejection after transplantation in a myocardial infarction in rats. ${ }^{76}$ However, immune rejection is still a major consideration particularly in the clinical trials of neurological diseases. Furthermore, immune suppression is still included in the protocols of clinical trials. ${ }^{77}$

3.2.2 Ethical issues. The utilization of ESCs triggers a lot of ethical debate as the cells for clinical use are obtained from aborted or deceased embryos. The legislatures in many 
Table 2 Major clinical trials investigating stem cells therapies for stroke treatment. Each trial is identified by its international registration code. The locations and testing stem cell types are also provided. Some of these trials have been reported as indicated while those that are yet not reported are indicated as "Pending". Those studies that finished without announcing any following up, including whether the data is under analysis, in the $\mathrm{NIH}$ database are indicated as "no information"

\begin{tabular}{|c|c|c|c|c|c|}
\hline & Trial ID & Country & Cell type & $\begin{array}{l}\text { Completion } \\
\text { date }\end{array}$ & Outcome \\
\hline 1 & NCT00473057 & Brazil & MSC: BMSC & 2011 & No information \\
\hline 3 & NCT00761982 & Spain & MSC: CD34+ & 2011 & Clinical safety ${ }^{74}$ \\
\hline 4 & NCT00535197 & UK & MSC: CD34+ & 2012 & No information \\
\hline 5 & NCT01297413 & USA & MSC: BMSC & 2013 & Pending \\
\hline 8 & NCT01678534 & Spain & MSC: adipose derived cells & 2013 & Clinical safety and efficacy ${ }^{75}$ \\
\hline 9 & NCT00859014 & USA & MSC: mononuclear cells & 2013 & Clinical safety ${ }^{66}$ \\
\hline 10 & NCT01028794 & Japan & Autologous BMMNC & 2013 & Clinical safety and efficacy ${ }^{70}$ \\
\hline 11 & NCT01518231 & PRC & Hematopoietic SC & 2013 & No information \\
\hline 12 & NCT01438593 & Taiwan & MSC: CD34+ & 2013 & Pending \\
\hline 13 & NCT02065778 & India & Autologous BMMNC & 2014 & Clinical safety ${ }^{67}$ \\
\hline 18 & NCT01436487 & USA \& UK & Multistem cells & 2014 & Pending \\
\hline 19 & NCT01287936 & USA & SB623 & 2015 & Pending \\
\hline 20 & NCT01849887 & USA & MSC: BMSC & 2015 & Pending \\
\hline 21 & NCT01714167 & PRC & MSC: BMSC & 2015 & Pending \\
\hline 22 & NCT01468064 & PRC & BMSC and EPC & 2015 & Pending \\
\hline 23 & NCT01716481 & Korea & MSC & 2016 & Pending \\
\hline 24 & NCT00875654 & France & MSC & 2016 & Pending \\
\hline
\end{tabular}

countries have discussed and established regulations regarding the research and clinical use of embryonic tissues. ${ }^{78}$ In order to avoid this conflict, iPSCs can serve as a suitable option as they not only reduce the risk of an immune response, but also circumvent any ethical conflicts. Similarly, autologous MSCs, currently highlighted as the main focus of stem cell research for stroke treatment, are another option that are not subject to the ethical debate because the stem cells originate from the recipient rather than a fetal or embryonic source..$^{79,80}$

3.2.3 Routes. The efficacy of stem cell transplantation is closely related to the route and location of grafting. Stem cells are directly and invasively applied into, or adjacent to, the infarcted region, but most studies are intravenous. For the safety of recipients, intravenous injection is a better approach while many studies adopt the peripheral transplantation of stem cells. ${ }^{55,64,68}$ This allows stem cells to grow and differentiate at locations near to the stroke penumbra. Although both endogenous and exogenous stem cells are capable of migrating to the affected regions, the efficiency of stem cell migration varies under different conditions. ${ }^{81}$ Instead of intracranial administration of stem cells, in which a craniotomy is usually needed, the delivery of stem cells into the circulation system, particularly for MSCs, provides a less invasive treatment option. $^{15,47}$ However, any experimental protocol must take precautions to prevent the formation of embolisms from the transplanted cells. ${ }^{82,83}$

3.2.4 Age. Age is a possible limiting factor in efficacious stem cell therapy. In most of the studies investigating stem cell therapies in animal models of stroke, young animals are usually utilized since it is methodologically easier. ${ }^{84}$ As most clinical cases of stroke occur in the elderly population however, it is more clinically realistic to use stroke models of aged animals. ${ }^{1}$ Complete spontaneous recovery from stroke can be observed in rat models using young subjects, while only minimal improvement is detected in aged rats. ${ }^{84}$ Furthermore, the pool of endogenous stem cells or precursor cells located in the SVZ is reduced by half in aged brains relative to young brains. ${ }^{85}$ This also implies that the environment in the brain of an aged rat is not particularly supportive for stem cell growth, which also results in a lower yield of functional stem cells.

\subsubsection{Critics of current research into stem cell therapies for} stroke treatment. Several systematic reviews with meta-analyses of stem cell therapies for ischemic stroke have been published based on both clinical and pre-clinical studies. ${ }^{72,73,86,87}$ Despite a robust and consistent improvement in the behavioral outcomes in pre-clinical studies, ${ }^{72}$ the meta-analyses indicate that the therapeutic effects of stem cell therapies in patients are modest, thus indicating a need to optimize the conditions of stem cell based approaches for stroke treatment. ${ }^{72}$ Further complicating the matter, clinical trials investigating stem cells are typically small and not randomized, thus casting doubt upon the validity of the findings. ${ }^{73,88}$ Randomized controlled trials on a larger scale are required to confirm the effectiveness of stem cell therapies for stroke. ${ }^{72}$

Most of the systematic reviews cited the difficulties in conducting these analyses due to the significant heterogeneity in 
those clinical studies. For instance, the difference types of stem cells used, with or without genetic modifications, different numbers of stem cells transplanted, varied locations of grafting, diverse baseline patient characteristics, measurement of outcomes, etc. make a fair comparison difficult to conduct. ${ }^{87}$

Furthermore, several systematic reviews were critical of the fact that most of the stem cell studies did not report graft cell survival in the brain and generally reported that cell therapymediated adverse events were not observed. These findings raise further concerns regarding publication bias. ${ }^{86}$

3.2.6 Measures to overcome the obstacles. There are numerous clinical trials that investigate the clinical therapeutic effects of different types of stem cells. Most of these demonstrate clinical safety and potential efficacy. However, some of the trials did not show efficacy in clinical treatment. Therefore, other approaches are needed to facilitate the therapeutic efficacy of stem cells in the treatment of stroke. As discussed previously, the use of iPSCs derived from the somatic cells harvested from the recipient of stem cell transplantation may eliminate the immune reactions and avoid ethical conflict. However, there are still no promising approaches to solving the problems relating to transplantation routes and the ages of the stroke patients. These issues affect the quality of stem cell growth and consequently hamper the promising therapeutic effects of transplanted stem cells. Accordingly, research into the applications of biomaterials is now gaining more attention in order to facilitate the growth of stem cells and develop a more reliable route for administration into damaged brains following an ischemic insult.

\section{Facilitation of stem cell therapy in stroke by tissue engineering and application of biomaterial}

A biomaterial, as defined by Prof. David Williams, is "a substance that has been engineered to take a form which, alone or as part of a complex system, is used to direct, by control of interactions with components of living systems, the course of any therapeutic or diagnostic procedure." ${ }^{89}$ Biomaterials have been used since the dawn of mankind. For example, nacre, also known as mother-of-pearl, was first used as a dental implant in the ancient Mayan civilization. ${ }^{90}$ The modern era of biomaterials started in the 1940s when Sir Harold Ridley used poly(methyl methacrylate) in intraocular lenses. ${ }^{91}$ Since that time, biomaterials have been applied successfully as scaffolds for tissue engineering and as delivery vehicles for drugs, proteins and genes. Since people have become aware of the problems associated with stem cell therapy, namely, low cell survival and inefficient neuronal cell differentiation, biomaterial-based scaffolds have been used to tackle such problems. To date, several biomaterials have been used (Table 3), including alginate, ${ }^{92}$ collagen, ${ }^{93}$ dextran, ${ }^{94}$ hyaluronan/methyl cellulose (HAMC), ${ }^{95,96}$ gelatin, ${ }^{97}$ and poly(lactic-co-glycolic acid) (PLGA). ${ }^{98-100}$ These biomaterials are either synthetic or natural polymers. Other types of biomaterials, such as ceramics and metals, are not suitable for brain tissue engineering due to the density and consistency of neural tissue. In this section, we will first address the desirable properties a biomaterial should possess for repairing or replacing brain tissue using six types of biomaterials as examples. Then we will look at the key properties of biomaterials that can influence stem cell proliferation and differentiation. Finally, we will describe the current strategy that combines two approaches, tissue engineering scaffolds and drug delivery vehicles, into one (Fig. 1).

\subsection{Desired biomaterials for brain tissue engineering}

The ideal biomaterial for use in brain tissue engineering should be biocompatible, biodegradable, should not generate an immune system reaction, and should not induce neurotoxicity. ${ }^{75}$ We will use six commonly used biomaterials as examples to illustrate these points.

4.1.1 Alginate. Alginate is a natural polymer that can be refined from brown seaweed. It contains two monomeric units, $\beta$-D-mannuronic acid (M) and $\alpha$-L-guluronic acid (G). It is anionic which allows it to easily form a hydrogel upon addition of divalent cations such as $\mathrm{Ca}^{2+}$. Alginate hydrogel has been extensively used in various biomedical applications including tissue engineering, cell therapy and drug delivery. ${ }^{101,102}$ Over the years, results in the literature have shown that alginate is biocompatible and biodegradable. In general, little or no immune response was detected towards alginate implants. ${ }^{103}$ However, high $\mathbf{M}$ content alginates have been shown to be immunogenic. ${ }^{\mathbf{1 0 4}}$ Regarding neurotoxicity, one study showed that alginate protects neurons against $\mathrm{H}_{2} \mathrm{O}_{2}$-induced neurotoxicity. ${ }^{105}$ Alginate hydrogel was used as a carrier in a system of sustained release of vascular endothelial growth factor (VEGF). The VEGF released from the hydrogel injected into the strokeinfarcted areas of the brains of rats reduced the strokeinduced asymmetric motion. ${ }^{92}$

4.1.2 Collagen. Collagen is the most abundant protein in mammals. Thus far, twenty-eight sub-types of collagen have been identified. ${ }^{\mathbf{1 0 6}}$ The most commonly used sub-type in biomaterials is collagen type I. Collagen has high hydroxyproline content and a typical triple helix structure. Collagen type I is also a major component of the extracellular matrix (ECM). Therefore, it can support cell attachment, cell spread and proliferation. It is biocompatible and biodegradable. Collagen products from animal sources are immunogenic. ${ }^{107,108}$ As collagen is a major component of the ECM, collagen-induced neurotoxicity is not expected. In a rat model of stroke transplanted with a complex of neural stem cells (NSCs) and collagen type I, neurological stroke severity for evaluation of the motor and sensory deficit of the rodents was significantly reduced and synaptogenesis was increased. ${ }^{93}$ Beneficial effects such as structural and functional recovery of the neural tissue were found.

4.1.3 Dextran. Dextran is a polysaccharide that can be used as a hydrogel ${ }^{109}$ as well as a microcarrier. ${ }^{110}$ GE healthcare produces two dextran-based microcarriers: Cytodex ${ }^{\circledR} 1$ and Cytodex® 3. Cytodex® 3 is a dextran microcarrier with a gelatin coating. Dextran is biocompatible ${ }^{\mathbf{1 1 1}}$ and biodegradable ${ }^{\mathbf{1 1 2}}$ (with proper modification). It is also not immunogenic or neurotoxic. 
Table 3 Biomaterials used in stem cell research. The biocompatibilities, biodegradabilities, immune reactions and neurotoxicities of the biomaterials investigated in neurological disease models are summarized

\begin{tabular}{|c|c|c|c|c|c|c|}
\hline Biomaterial & Origin & Biocompatible & Biodegradable & $\begin{array}{l}\text { Major immune } \\
\text { response }\end{array}$ & Neurotoxicity & Key reference \\
\hline Alginate & Natural & Yes & Yes & No & No & 101 and 105 \\
\hline Dextran & Natural & Yes & Yes & No & No & 109 and 114 \\
\hline Hyaluronan/methyl cellulose (HAMC) & Hybrid & Yes & Yes & No & No & 95 and 115 \\
\hline Gelatin & Natural & Yes & Yes & Yes & No & 97 \\
\hline
\end{tabular}

a)

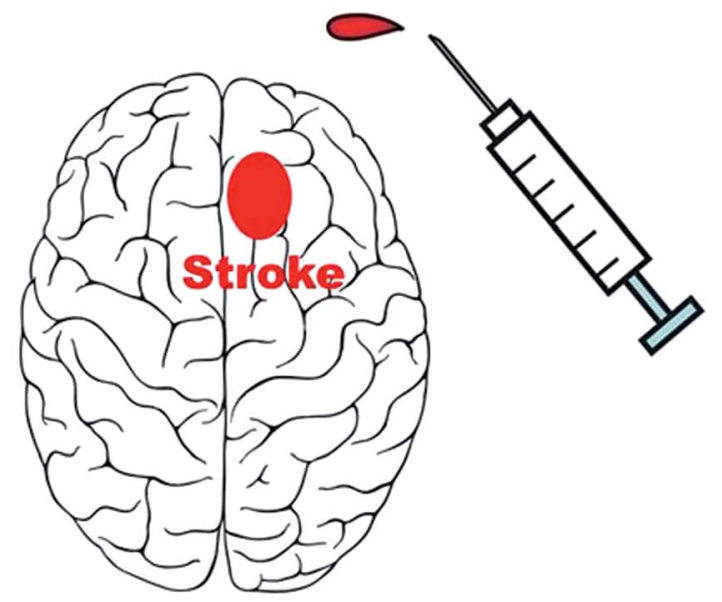

b)

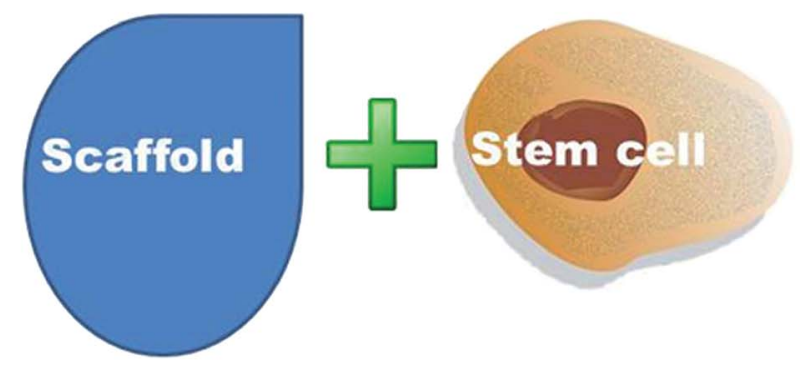

Fig. 1 Facilitation of stem cell therapy in stroke by tissue engineering and the application of biomaterials. (a) Schematic showing the transplantation of stem cells into the brain; (b) stem cells can be either encapsulated into microcapsules or cultivated on microspheres before implantation. Both microcapsules and microspheres are tissue engineering scaffolds made of alginate, collagen or other biomaterials.

Dextran has a long history in the treatment of stroke dating back to the 1960s. ${ }^{113}$ Infusion of dextran 40 (Rheomacrodex, hydrogel form) exhibited beneficial effects in patients; embolization in the peripheral circulation is highly eliminated. ${ }^{114}$ In another study, dextran-based microcarriers (Cytodex® 3 ) were used as scaffolds for rat adrenal chromaffin cells capable of secreting dopamine. ${ }^{94}$ The cell-attached dextran beads were implanted into the brains of rats and beneficial effects were observed.
4.1.4 Hyaluronan/methyl cellulose (HAMC). HAMC is a blend of hyaluronan (HA) and methyl cellulose (MC). HAMC is a hydrogel developed by Molly S. Shoichet's group at the University of Toronto. ${ }^{115}$ This material is unique compared to other hydrogel materials because it is fast-gelling and injectable. Up to now, results in the literature have demonstrated that this material is biocompatible and biodegradable. ${ }^{95}$ It also does not generate major immune reactions nor does it induce neurotoxicity. One study showed that HAMC administered as a drug delivery carrier for erythropoeitin (EPO) in a rat model of stroke resulted in the reduction of infarct volume, elimination of astrocytic and microglial activation, and promotion of neurogenesis. ${ }^{95}$ Interestingly, HAMC treated alone without EPO also produced a similar therapeutic effect.

4.1.5 Gelatin. Gelatin is a denatured form of collagen. It is biocompatible, biodegradable and does not induce neurotoxicity. Similar to collagen, it is questionable whether gelatin derived from animal sources should be used as it could cause some immune system reactions, such as allergy. Gelatin-based microcarriers have been used as cell culture scaffolds. Human retinal pigment epithelial (hRPE) cells that can be used as workhorses to generate L-DOPA and hRPE-conjugated gelatin beads have been implanted into patients with Parkinson's disease. $^{96}$

4.1.6 Poly(lactic-co-glycolic acid) (PLGA). PLGA is a synthetic polymer and has been approved by the US FDA for use as a drug carrier. It is biocompatible, biodegradable and does not induce neurotoxicity. PLGA has shown immunogenicity in BalB/c mice. ${ }^{116}$ PLGA is typically used as a microcarrier for drug delivery. ${ }^{99}$ Furthermore, PLGA beads have been used as scaffolds for stem cells. ${ }^{98}$

\subsection{Key factors that influence stem cell proliferation and differentiation}

We have discussed six major types of commonly used biomaterials for brain tissue engineering. Not only should the biomaterials facilitate the delivery of the substance for stroke treatment, but they should also provide a suitable medium for the growth and delivery of stem cells. To promote stem cell proliferation and differentiation, we can further adjust the properties of the materials. In the following section, we will cover four major properties of biomaterials: material size and shape, surface charge and hydrophobicity, surface chemistry and ligand/ receptors, and mechano-regulation/material elasticity. 
4.2.1 Material size and shape. The sizes and shapes of the materials play an important role in the stem cell-material interactions. For example, the sizes of microcarriers used as scaffolds for cell culture are critical. Earlier studies showed that selecting the appropriate microsphere diameter could affect cell growth kinetics in a bioreactor. ${ }^{\mathbf{1 1 7}}$ In addition, for brain tissue engineering, smaller sized scaffolds can be easily implanted into the brain. For example, Cytodex® 3 has a diameter of approximately $175 \mu \mathrm{m},{ }^{94}$ whereas gelatin microcarriers have diameters of around $100 \mu \mathrm{m} .{ }^{97}$ Both have been successfully injected into brain tissue. Besides the effect of size, nanotopography has also been shown to be able to guide mesenchymal stem cells towards neuronal differentiation. ${ }^{118}$ Recently, another study showed that nanogrooved substrates promoted the direct lineage reprogramming of somatic fibroblasts to induced dopaminergic (iDA) neurons. ${ }^{119}$ Joseph M. DeSimone's group at the University of North Carolina has systematically studied the cellular internalization of micro- and nanoparticles by controlling size, shape and surface chemistry of the particles. ${ }^{120}$ They found that under the conditions and maintaining the same surface chemistry, the size and shape of the particles plays a very important role.

4.2.2 Surface charge and hydrophobicity. The surface charge and hydrophobicity of the implanted biomaterials also play an important role. ${ }^{121}$ As early as the 1980s, scientists had found that under high shear stress, more HeLa cells attached to microcarriers with positively charged surfaces compared to microcarriers with negatively charged surfaces. ${ }^{122}$ In an in vitro DNA release study, researchers found that hydrophilic PLGA microspheres exhibited higher DNA encapsulation efficiency and a faster release rate in comparison with hydrophobic microspheres. ${ }^{123}$ In another study, the hydrophobicity of a series of polymeric films was carefully tuned in order to understand the influence of surface characteristics on cellular response. ${ }^{\mathbf{1 2 4}} \mathrm{A}$ difference in cell adhesion and growth was clearly seen.

4.2.3 Surface chemistry and ligands. Surface chemistry, especially coating a surface with various extracellular matrix (ECM) proteins, has a great impact on stem cell proliferation and differentiation. ${ }^{125}$ For example, laminin is known to help stem cells undergo neuronal differentiation. Laminin and fibronectin coated scaffolds improved stem cell survival after implantation. ${ }^{\mathbf{1 2 6}}$ Surface-coated polydopamine enhanced human neural stem cell differentiation and proliferation. ${ }^{\mathbf{1 2 7}}$ Kristi S. Anseth's group at the University of Colorado has shown that small-molecule functional groups tethered to hydrogels can control human mesenchymal stem cell differentiation into a certain lineage. ${ }^{\mathbf{1 2 8}}$

4.2.4 Mechano-regulation/material elasticity. In the final part of this section, we will discuss the importance of the mechanical properties of materials on stem cell differentiation. Dennis E. Discher's group at the University of Pennsylvania originally pointed out that "Matrix elasticity directs stem cell lineage specification". ${ }^{129}$ Ever since their publication in Cell in 2006, over five thousand papers have been published aiming to prove their theory. Among those papers, in one study, inkjet printing (recreating a digital image by propelling droplets of ink onto paper, plastic or other substrates) of hydrogels encapsulated with neural stem cells was explored. ${ }^{\mathbf{1 3 0}}$ Their results showed that neural stem cells favored neural lineages. 3D printing (additive manufacturing) provides us with another dimension for stem cell therapy.

\subsection{Application of biomaterials in stem cell transplantation for stroke therapy}

Stem cells have been transplanted into the brains of stroked animals, with stunning therapeutic effect, ${ }^{15,35,36,39,45,46}$ and mixed outcomes have also been observed in preliminary clinical cases. ${ }^{15,62}$ However, the survival rate and disorientation of stem cell differentiation are still major obstacles to guaranteeing the potential for clinical use. Applying biomaterials that have been shown to be beneficial for stem cell growth could increase the potency of stem cell treatment of stroke, as shown in a few successful examples. For instance, although there was no behavioral data to support the symptom-relieving effect, transplantation of neural stem cells supported with a PLGA scaffold reduced the ischemic lesion volume in the cerebral cortex in a rat MCAO model. ${ }^{98}$ As mentioned, Yu et al. successfully proved that administration of a complex containing collagen type I and neural stem cells can reduce the neurological stroke score in a rat MCAO model. ${ }^{93}$ Another phenomenal finding presented by Jin et al. showed that post-stroke administration of human neural precursor cells supported by matrigel not only reduced the infarct volume in the cerebral cortex following MCAO stroke, but also improved the preferred usage of the healthy limb only in the cylinder test and the learning and memory deficit in the Y-maze. ${ }^{\mathbf{1 3 1}}$ Although there are only several successful reports on the therapeutic effect of combined usage of stem cells and biomaterials, it is expected that more reports will be published. These findings strongly suggest that the combination of biomaterials and stem cells can provide a better therapeutic effect for lesioned animal stroke models than stem cell therapies in isolation.

\subsection{Combining cell scaffolds and drug carriers into stem cell therapies for stroke}

Pharmacologically active microcarrier (PAM) is an example of combining a cell scaffold and a drug carrier into one. ${ }^{\mathbf{1 0 0}}$ In a recent study, mesenchymal stem cells and VEGF were loaded into PAM. ${ }^{100}$ It has been shown to be an effective strategy for repairing the brain damage caused by stroke. PAM was initially developed by Claudia N. Montero-Menei's group at INSERM, France. ${ }^{132}$ It serves as a support for cell culture and a carrier for the controlled delivery of bioactive molecules. In addition to stroke, Claudia N. Montero-Menei's group also investigated the use of PAM to treat Parkinson's disease ${ }^{\mathbf{1 3 3}}$ and osteoarthritis. ${ }^{\mathbf{1 3 4}}$ PAM is based on PLGA microcarriers. Other types of biomaterials can be used and even better outcomes are expected to be achieved using the same strategy but with different types of microcarriers. In addition, a limited number of growth factors have been tested, such as VEGF ${ }^{104}$ and TGF- $\beta .^{134}$ Further research into the therapeutic effects of a broader array of growth 
factors will be carried out in the near future. A combination of two or more growth factors can be used to achieve much better results for stem cell therapy.

\section{Conclusion}

Stem cell therapy in stroke is in its infant stage. There are still numerous technical problems and obstacles yet to be solved. Biomaterial based tissue engineering is a promising approach for facilitation of the potency of transplanted stem cells in stroke treatment. However, the safety and biocompatibility of biomaterials are major issues to be considered in order to develop a more promising therapeutic approach for clinical use in stroke therapy. Furthermore, most of the research concerning the combination of biomaterials with stem cell transplantation in in vivo stroke models focuses mainly on cell survival, differentiation, and localization with limited data comparing behavioral outcomes, which have not been clinically reviewed. Another important issue that is worthy of attention is that transplanted stem cells cannot guarantee the formation of the desired neural circuit for functional neurorestoration following stroke. Further investigation is necessary in order to ensure that transplanted stem cells can re-integrate with functional circuitry. Undeniably, stem cell therapy will be developed into a safe and reliable treatment for restoration of brain functions from any form of brain injury, particularly stroke. We expect that more works on stem cell therapy development, including clinical trials, will be published in the near future.

\section{Acknowledgements}

S. Z. thanks the National High Technology Research and Development Program of China (2015AA020508), the National Key Basic Research Program of China (2015CB352006), the National Natural Science Foundation of China (61335011), the Program for Changjiang Scholars and Innovative Research Team in University (Grant No. IRT_15R10), and the Fujian Provincial Youth Top-notch Talent Support Program.

\section{References}

1 V. L. Roger, A. S. Go and D. M. Lloyd-Jones, et al., Heart disease and stroke statistics - 2012 update: a report from the American Heart Association. Circulation, Jan 3 2012, vol. 125, 1, pp. e2-e220.

2 F. Gokcay, E. M. Arsava, T. Baykaner, et al., Age-dependent susceptibility to infarct growth in women, Stroke, 2011, 42(4), 947-951.

3 M. Mott, K. Pahigiannis and W. Koroshetz, Small blood vessels: big health problems: National Institute of Neurological Disorders and Stroke update, Stroke, 2014, 45(12), e257-258.

4 J. E. Jung, G. S. Kim, H. Chen, et al., Reperfusion and neurovascular dysfunction in stroke: from basic mechanisms to potential strategies for neuroprotection, Mol. Neurobiol., 2010, 41(2-3), 172-179.
5 A. Brassai, R. G. Suvanjeiev, E. G. Ban and M. Lakatos, Role of synaptic and nonsynaptic glutamate receptors in ischaemia induced neurotoxicity, Brain Res. Bull., 2015, 112, 1-6.

6 A. Pollock, S. E. Farmer, M. C. Brady, et al., Interventions for improving upper limb function after stroke, Cochrane Database of Systematic Reviews, 2014, 11, CD010820.

7 K. M. Langa and D. A. Levine, The diagnosis and management of mild cognitive impairment: a clinical review, J. Am. Med. Assoc., 2014, 312(23), 2551-2561.

8 J. H. Sun, L. Tan and J. T. Yu, Post-stroke cognitive impairment: epidemiology, mechanisms and management, Ann. Transl. Med., 2014, 2(8), 80.

9 A. De Ryck, R. Brouns, M. Geurden, M. Elseviers, P. P. De Deyn and S. Engelborghs, Risk factors for poststroke depression: identification of inconsistencies based on a systematic review, J. Geriatr. Psychiatr. Neurol., 2014, 27(3), 147-158.

10 A. J. Wu, J. Radel and B. Hanna-Pladdy, Improved function after combined physical and mental practice after stroke: a case of hemiparesis and apraxia, Am. J. Occup. Ther., 2011, 65(2), 161-168.

11 F. Malouin, S. Belleville, C. L. Richards, J. Desrosiers and J. Doyon, Working memory and mental practice outcomes after stroke, Arch. Phys. Med. Rehabil., 2004, 85(2), 177-183.

12 R. M. Appireddy, A. M. Demchuk, M. Goyal, et al., Endovascular therapy for ischemic stroke, J. Clin. Neurol., 2015, 11(1), 1-8.

13 S. Yaghi, A. Eisenberger and J. Z. Willey, Symptomatic intracerebral hemorrhage in acute ischemic stroke after thrombolysis with intravenous recombinant tissue plasminogen activator: a review of natural history and treatment, JAMA Neurol., 2014, 71(9), 1181-1185.

14 K. Abe, T. Yamashita, S. Takizawa, S. Kuroda, H. Kinouchi and N. Kawahara, Stem cell therapy for cerebral ischemia: from basic science to clinical applications, J. Cereb. Blood Flow Metab., 2012, 32(7), 1317-1331.

15 T. Honma, O. Honmou, S. Iihoshi, et al., Intravenous infusion of immortalized human mesenchymal stem cells protects against injury in a cerebral ischemia model in adult rat, Exp. Neurol., 2006, 199(1), 56-66.

16 S. I. Savitz, M. Chopp, R. Deans, T. Carmichael, D. Phinney and L. Wechsler, Stem Cell Therapy as an Emerging Paradigm for Stroke (STEPS) II, Stroke, 2011, 42(3), 825-829.

17 P. Thored, A. Arvidsson, E. Cacci, et al., Persistent production of neurons from adult brain stem cells during recovery after stroke, Stem Cells, 2006, 24(3), 739-747.

18 M. K. Tobin, J. A. Bonds, R. D. Minshall, D. A. Pelligrino, F. D. Testai and O. Lazarov, Neurogenesis and inflammation after ischemic stroke: what is known and where we go from here, J. Cereb. Blood Flow Metab., 2014, 34(10), 1573-1584.

19 T. Talwar and M. V. Srivastava, Role of vascular endothelial growth factor and other growth factors in post-stroke recovery, Ann. Indian Acad. Neurol., 2014, 17(1), 1-6.

20 M. Slevin, J. Krupinski, N. Rovira, et al., Identification of pro-angiogenic markers in blood vessels from stroked- 
affected brain tissue using laser-capture microdissection, BMC Genomics, 2009, 10, 113.

21 J. Macas, C. Nern, K. H. Plate and S. Momma, Increased generation of neuronal progenitors after ischemic injury in the aged adult human forebrain, J. Neurosci., 2006, 26(50), 13114-13119.

22 S. L. Minger, A. Ekonomou, E. M. Carta, A. Chinoy, R. H. Perry and C. G. Ballard, Endogenous neurogenesis in the human brain following cerebral infarction, Regener. Med., 2007, 2(1), 69-74.

$23 \mathrm{~J}$. M. Parent, Injury-induced neurogenesis in the adult mammalian brain, Neuroscientist, 2003, 9(4), 261-272.

24 M. J. Zaben and W. P. Gray, Neuropeptides and hippocampal neurogenesis, Neuropeptides, 2013, 47(6), 431-438.

25 M. S. Kim, H. R. Park, M. Park, et al., Neurotoxic effect of 2,5-hexanedione on neural progenitor cells and hippocampal neurogenesis, Toxicology, 2009, 260(1-3), 97-103.

26 M. Komitova, B. Mattsson, B. B. Johansson and P. S. Eriksson, Enriched environment increases neural stem/progenitor cell proliferation and neurogenesis in the subventricular zone of stroke-lesioned adult rats, Stroke, 2005, 36(6), 1278-1282.

27 H. Y. Zhou, Y. Katsman, N. K. Dhaliwal, et al., A Sox2 distal enhancer cluster regulates embryonic stem cell differentiation potential, Genes Dev., 2014, 28(24), 26992711.

28 J. J. Ohab and S. T. Carmichael, Poststroke neurogenesis: emerging principles of migration and localization of immature neurons, Neuroscientist, 2008, 14(4), 369-380.

29 C. R. Tyler and A. M. Allan, Adult hippocampal neurogenesis and mRNA expression are altered by perinatal arsenic exposure in mice and restored by brief exposure to enrichment, PLoS One, 2013, 8(9), e73720.

30 J. F. Arenillas, T. Sobrino, J. Castillo and A. Davalos, The role of angiogenesis in damage and recovery from ischemic stroke, Curr. Treat. Options Cardiovasc. Med., 2007, 9(3), 205-212.

31 T. G. Liman and M. Endres, New vessels after stroke: postischemic neovascularization and regeneration, Cerebrovasc. Dis., 2012, 33(5), 492-499.

32 G. Bain, D. Kitchens, M. Yao, J. E. Huettner and D. I. Gottlieb, Embryonic stem cells express neuronal properties in vitro, Dev. Biol., 1995, 168(2), 342-357.

33 S. Okabe, K. Forsberg-Nilsson, A. C. Spiro, M. Segal and R. D. McKay, Development of neuronal precursor cells and functional postmitotic neurons from embryonic stem cells in vitro, Mech. Dev., 1996, 59(1), 89-102.

34 S. C. Zhang, M. Wernig, I. D. Duncan, O. Brustle and J. A. Thomson, In vitro differentiation of transplantable neural precursors from human embryonic stem cells, Nat. Biotechnol., 2001, 19(12), 1129-1133.

35 L. Wei, L. Cui, B. J. Snider, et al., Transplantation of embryonic stem cells overexpressing Bcl-2 promotes functional recovery after transient cerebral ischemia, Neurobiol. Dis., 2005, 19(1-2), 183-193.
36 D. Yanagisawa, M. Qi, D. H. Kim, et al., Improvement of focal ischemia-induced rat dopaminergic dysfunction by striatal transplantation of mouse embryonic stem cells, Neurosci. Lett., 2006, 407(1), 74-79.

37 D. Solter, From teratocarcinomas to embryonic stem cells and beyond: a history of embryonic stem cell research, Nat. Rev. Genet., 2006, 7(4), 319-327.

38 O. Lindvall and Z. Kokaia, Stem cell research in stroke: how far from the clinic?, Stroke, 2011, 42(8), 2369-2375.

39 K. Takahashi, T. Yasuhara, T. Shingo, et al., Embryonic neural stem cells transplanted in middle cerebral artery occlusion model of rats demonstrated potent therapeutic effects, compared to adult neural stem cells, Brain Res., 2008, 1234, 172-182.

40 A. J. Friedenstein, S. Piatetzky and K. V. Petrakova II, Osteogenesis in transplants of bone marrow cells, $J$. Embryol. Exp. Morphol., 1966, 16(3), 381-390.

41 Z. Zou, Y. Zhang, L. Hao, et al., More insight into mesenchymal stem cells and their effects inside the body, Expert Opin. Biol. Ther., 2010, 10(2), 215-230.

42 K. M. Sicard and M. Fisher, Animal models of focal brain ischemia, Exp. Transl. Stroke Med., 2009, 1, 7.

43 G. Gilmour, S. D. Iversen, M. F. O'Neill and D. M. Bannerman, The effects of intracortical endothelin1 injections on skilled forelimb use: implications for modelling recovery of function after stroke, Behav. Brain Res., 2004, 150(1-2), 171-183.

44 M. F. Pittenger, A. M. Mackay, S. C. Beck, et al., Multilineage potential of adult human mesenchymal stem cells, Science, 1999, 284(5411), 143-147.

45 K. Kurozumi, K. Nakamura, T. Tamiya, et al., BDNF genemodified mesenchymal stem cells promote functional recovery and reduce infarct size in the rat middle cerebral artery occlusion model, Mol. Ther., 2004, 9(2), 189-197.

46 D. C. Ding, W. C. Shyu, M. F. Chiang, et al., Enhancement of neuroplasticity through upregulation of beta1-integrin in human umbilical cord-derived stromal cell implanted stroke model, Neurobiol. Dis., 2007, 27(3), 339-353.

47 Y. Omori, O. Honmou, K. Harada, J. Suzuki, K. Houkin and J. D. Kocsis, Optimization of a therapeutic protocol for intravenous injection of human mesenchymal stem cells after cerebral ischemia in adult rats, Brain Res., 2008, 1236, 30-38.

48 X. Bao, M. Feng, J. Wei, et al., Transplantation of Flk-1+ human bone marrow-derived mesenchymal stem cells promotes angiogenesis and neurogenesis after cerebral ischemia in rats, Eur. J. Neurosci., 2011, 34(1), 87-98.

49 S. Suda, K. I. Katsura, M. Saito, N. Kamiya and Y. Katayama, Valproic acid enhances the effect of bone marrow-derived mononuclear cells in a rat ischemic stroke model, Brain Res., 2014, 1565, 74-81.

50 Y. Li, W. W. Mao, C. G. Zhang, et al., Neuroprotective effects of intravenous transplantation of bone marrow mononuclear cells from 5-fluorouracil pre-treated rats on ischemic stroke, Behav. Brain Res., 2016, 301, 287-292.

51 M. Song, S. S. Jue, Y. A. Cho and E. C. Kim, Comparison of the effects of human dental pulp stem cells and human 
bone marrow-derived mesenchymal stem cells on ischemic human astrocytes in vitro, J. Neurosci. Res., 2015, 93(6), 973983.

52 M. Song, J. H. Lee, J. Bae, Y. Bu and E. C. Kim, Human dental pulp stem cells are more effective than human bone marrowderived mesenchymal stem cells in cerebral ischemic injury, Cell Transplant., 2017, DOI: 10.3727/096368916X694391.

53 G. Varga and G. Gerber, Mesenchymal stem cells of dental origin as promising tools for neuroregeneration, Stem Cell Res. Ther., 2014, 5(2), 61.

54 B. Mitkari, E. Kerkela, J. Nystedt, et al., Intra-arterial infusion of human bone marrow-derived mesenchymal stem cells results in transient localization in the brain after cerebral ischemia in rats, Exp. Neurol., 2013, 239, 158-162.

55 M. Gutierrez-Fernandez, B. Rodriguez-Frutos, J. RamosCejudo, et al., Effects of intravenous administration of allogenic bone marrow- and adipose tissue-derived mesenchymal stem cells on functional recovery and brain repair markers in experimental ischemic stroke, Stem Cell Res. Ther., 2013, 4(1), 11.

56 E. M. Pietras, M. R. Warr and E. Passegue, Cell cycle regulation in hematopoietic stem cells, J. Cell Biol., 2011, 195(5), 709-720.

57 H. Felfly, A. Muotri, H. Yao and G. G. Haddad, Hematopoietic stem cell transplantation protects mice from lethal stroke, Exp. Neurol., 2010, 225(2), 284-293.

58 D. Doycheva, G. Shih, H. Chen, R. Applegate, J. H. Zhang and J. Tang, Granulocyte-colony stimulating factor in combination with stem cell factor confers greater neuroprotection after hypoxic-ischemic brain damage in the neonatal rats than a solitary treatment, Transl. Stroke Res., 2013, 4(2), 171-178.

$59 \mathrm{H}$. Okano and S. Yamanaka, iPS cell technologies: significance and applications to CNS regeneration and disease, Mol. Brain., 2014, 7, 22.

60 S. J. Chen, C. M. Chang, S. K. Tsai, et al., Functional improvement of focal cerebral ischemia injury by subdural transplantation of induced pluripotent stem cells with fibrin glue, Stem Cells Dev., 2010, 19(11), 17571767.

61 M. Jiang, L. Lv, H. Ji, et al., Induction of pluripotent stem cells transplantation therapy for ischemic stroke, Mol. Cell. Biochem., 2011, 354(1-2), 67-75.

62 D. Kalladka and K. W. Muir, Brain repair: cell therapy in stroke, Stem Cells Cloning., 2014, 7, 31-44.

63 F. Bifari, L. Pacelli and M. Krampera, Immunological properties of embryonic and adult stem cells, World $J$. Stem Cells., 2010, 2(3), 50-60.

64 O. Honmou, K. Houkin, T. Matsunaga, et al., Intravenous administration of auto serum-expanded autologous mesenchymal stem cells in stroke, Brain., 2011, 134(Pt 6), 1790-1807.

65 A. Bhasin, M. V. Srivastava, S. Mohanty, R. Bhatia, S. S. Kumaran and S. Bose, Stem cell therapy: a clinical trial of stroke, Clin Neurol Neurosurg., 2013, 115(7), 10031008.
66 S. I. Savitz, V. Misra, M. Kasam, et al., Intravenous autologous bone marrow mononuclear cells for ischemic stroke, Ann Neurol., 2011, 70(1), 59-69.

67 A. Sharma, H. Sane, N. Gokulchandran, et al., Autologous bone marrow mononuclear cells intrathecal transplantation in chronic stroke, Stroke Res. Treat., 2014, 2014, 234095.

68 K. Prasad, S. Mohanty, R. Bhatia, et al., Autologous intravenous bone marrow mononuclear cell therapy for patients with subacute ischaemic stroke: a pilot study, Indian J. Med. Res., 2012, 136(2), 221-228.

69 K. Prasad, A. Sharma, A. Garg, et al., Intravenous autologous bone marrow mononuclear stem cell therapy for ischemic stroke: a multicentric, randomized trial, Stroke., 2014, 45(12), 3618-3624.

70 A. Taguchi, C. Sakai, T. Soma, et al., Intravenous Autologous Bone Marrow Mononuclear Cell Transplantation for Stroke: Phase1/2a Clinical Trial in a Homogeneous Group of Stroke Patients, Stem Cells Dev., 2015, 24(19), 2207-2218.

71 G. H. Petit, T. T. Olsson and P. Brundin, The future of cell therapies and brain repair: Parkinson's disease leads the way, Neuropathol Appl Neurobiol., 2014, 40(1), 60-70.

72 Q. Vu, K. Xie, M. Eckert, W. Zhao and S. C. Cramer, Metaanalysis of preclinical studies of mesenchymal stromal cells for ischemic stroke, Neurology., 2014, 82(14), 12771286.

73 H. Jeong, H. W. Yim, Y. S. Cho, et al., Efficacy and safety of stem cell therapies for patients with stroke: a systematic review and single arm meta-analysis, Int J Stem Cells., 2014, 7(2), 63-69.

74 F. Moniche, A. Gonzalez, J. R. Gonzalez-Marcos, et al., Intraarterial bone marrow mononuclear cells in ischemic stroke: a pilot clinical trial, Stroke., 2012, 43(8), 2242-2244.

75 E. Diez-Tejedor, M. Gutierrez-Fernandez, P. MartinezSanchez, et al., Reparative therapy for acute ischemic stroke with allogeneic mesenchymal stem cells from adipose tissue: a safety assessment: a phase II randomized, double-blind, placebo-controlled, singlecenter, pilot clinical trial, J. Stroke Cerebrovasc Dis., 2014, 23(10), 2694-2700.

76 Y. Imanishi, A. Saito, H. Komoda, et al., Allogenic mesenchymal stem cell transplantation has a therapeutic effect in acute myocardial infarction in rats, J. Mol. Cell. Cardiol., 2008, 44(4), 662-671.

77 J. R. Evans, S. L. Mason and R. A. Barker, Current status of clinical trials of neural transplantation in Parkinson's disease, Prog. Brain Res., 2012, 200, 169-198.

$78 \mathrm{~N}$. Lenoir, Europe confronts the embryonic stem cell research challenge, Science, 2000, 287(5457), 1425-1427.

79 I. Kalaszczynska and K. Ferdyn, Wharton's jelly derived mesenchymal stem cells: future of regenerative medicine? Recent findings and clinical significance, Biomed Res Int., 2015, 2015, 430847.

80 A. Hilfiker, C. Kasper, R. Hass and A. Haverich, Mesenchymal stem cells and progenitor cells in connective tissue engineering and regenerative medicine: 
is there a future for transplantation?, Langenbecks Arch. Surg., 2011, 396(4), 489-497.

81 S. Kelly, T. M. Bliss, A. K. Shah, et al., Transplanted human fetal neural stem cells survive, migrate, and differentiate in ischemic rat cerebral cortex, Proc. Natl. Acad. Sci. U. S. A., 2004, 101(32), 11839-11844.

82 J. W. Jung, M. Kwon, J. C. Choi, et al., Familial occurrence of pulmonary embolism after intravenous, adipose tissuederived stem cell therapy, Yonsei Med J., 2013, 54(5), 1293-1296.

83 T. Makela, R. Takalo, O. Arvola, et al., Safety and biodistribution study of bone marrow-derived mesenchymal stromal cells and mononuclear cells and the impact of the administration route in an intact porcine model, Cytotherapy., 2015, 17(4), 392-402.

84 A. Popa-Wagner, A. M. Buga, T. R. Doeppner and D. M. Hermann, Stem cell therapies in preclinical models of stroke associated with aging, Front Cell Neurosci., 2014, 8, 347.

85 E. Enwere, T. Shingo, C. Gregg, H. Fujikawa, S. Ohta and S. Weiss, Aging results in reduced epidermal growth factor receptor signaling, diminished olfactory neurogenesis, and deficits in fine olfactory discrimination, J. Neurosci., 2004, 24(38), 8354-8365.

86 J. S. Lees, E. S. Sena, K. J. Egan, et al., Stem cell-based therapy for experimental stroke: a systematic review and meta-analysis, Int J Stroke., 2012, 7(7), 582-588.

87 W. Cao and P. Li, Effectiveness and Safety of Autologous Bone Marrow Stromal Cells Transplantation After Ischemic Stroke: A Meta-Analysis, Med. Sci. Monit., 2015, 21, 2190-2195.

88 G. B. Boncoraglio, A. Bersano, L. Candelise, B. A. Reynolds and E. A. Parati, Stem cell transplantation for ischemic stroke, Cochrane Database of Systematic Reviews, 2010, (9), CD007231.

89 D. F. Williams, On the mechanisms of biocompatibility, Biomaterials, 2008, 29(20), 2941-2953.

$90 \mathrm{M}$. Ni and B. D. Ratner, Nacre surface transformation to hydroxyapatite in a phosphate buffer solution, Biomaterials, 2003, 24(23), 4323-4331.

91 B. D. Ratner and S. J. Bryant, Biomaterials: where we have been and where we are going, Annu. Rev. Biomed. Eng., 2004, 6, 41-75.

92 D. F. Emerich, E. Silva, O. Ali, et al., Injectable VEGF hydrogels produce near complete neurological and anatomical protection following cerebral ischemia in rats, Cell Transplant., 2010, 19(9), 1063-1071.

$93 \mathrm{H}$. Yu, B. Cao, M. Feng, et al., Combinated transplantation of neural stem cells and collagen type I promote functional recovery after cerebral ischemia in rats, Anat. Rec., 2010, 293(5), 911-917.

94 B. D. Cherksey, V. S. Sapirstein and A. L. Geraci, Adrenal chromaffin cells on microcarriers exhibit enhanced longterm functional effects when implanted into the mammalian brain, Neuroscience, 1996, 75(2), 657-664.

95 Y. Wang, M. J. Cooke, C. M. Morshead and M. S. Shoichet, Hydrogel delivery of erythropoietin to the brain for endogenous stem cell stimulation after stroke injury, Biomaterials, 2012, 33(9), 2681-2692.

96 M. J. Cooke, Y. Wang, C. M. Morshead and M. S. Shoichet, Controlled epi-cortical delivery of epidermal growth factor for the stimulation of endogenous neural stem cell proliferation in stroke-injured brain, Biomaterials, 2011, 32(24), 5688-5697.

97 N. P. Stover and R. L. Watts, Spheramine for treatment of Parkinson's disease, Neurotherapeutics, 2008, 5(2), 252-259.

98 E. Bible, D. Y. Chau, M. R. Alexander, J. Price, K. M. Shakesheff and M. Modo, The support of neural stem cells transplanted into stroke-induced brain cavities by PLGA particles, Biomaterials, 2009, 30(16), 2985-2994.

99 E. Bible, O. Qutachi, D. Y. Chau, M. R. Alexander, K. M. Shakesheff and M. Modo, Neo-vascularization of the stroke cavity by implantation of human neural stem cells on VEGF-releasing PLGA microparticles, Biomaterials, 2012, 33(30), 7435-7446.

100 M. S. Quittet, O. Touzani, L. Sindji, et al., Effects of mesenchymal stem cell therapy, in association with pharmacologically active microcarriers releasing VEGF, in an ischaemic stroke model in the rat, Acta Biomater., $2015,15,77-88$.

101 K. Y. Lee and D. J. Mooney, Alginate: properties and biomedical applications, Prog. Polym. Sci., 2012, 37(1), 106-126.

102 A. D. Augst, H. J. Kong and D. J. Mooney, Alginate hydrogels as biomaterials, Macromol. Biosci., 2006, 6(8), 623-633.

103 U. Zimmermann, G. Klock, K. Federlin, et al., Production of mitogen-contamination free alginates with variable ratios of mannuronic acid to guluronic acid by free flow electrophoresis, Electrophoresis, 1992, 13(5), 269-274.

104 M. Otterlei, K. Ostgaard, G. Skjak-Braek, O. Smidsrod, P. Soon-Shiong and T. Espevik, Induction of cytokine production from human monocytes stimulated with alginate, J. Immunother., 1991, 10(4), 286-291.

105 B. Eftekharzadeh, F. Khodagholi, A. Abdi and N. Maghsoudi, Alginate protects NT2 neurons against $\mathrm{H}_{2} \mathrm{O}_{2}$-induced neurotoxicity, Carbohydr. Polym., 2010, 79(4), 1063-1072.

106 L. Cen, W. Liu, L. Cui, W. Zhang and Y. Cao, Collagen tissue engineering: development of novel biomaterials and applications, Pediatr. Res., 2008, 63(5), 492-496.

107 C. Soo, G. Rahbar and R. L. Moy, The immunogenicity of bovine collagen implants, Dermatol. Surg., 1993, 19(5), 431-434.

108 A. K. Lynn, I. V. Yannas and W. Bonfield, Antigenicity and immunogenicity of collagen, J. Biomed. Mater. Res., Part B, 2004, 71(2), 343-354.

109 J. A. Cadee, M. J. van Luyn, L. A. Brouwer, et al., In vivo biocompatibility of dextran-based hydrogels, J. Biomed. Mater. Res., 2000, 50(3), 397-404.

110 C. Gebb, B. Lundgren, J. Clark and U. Lindskog, Harvesting and subculturing cells growing on denatured-collagen coated microcarriers (Cytodex 3), Dev. Biol. Stand., 1983, 55, 57-65. 
111 C. J. De Groot, M. J. Van Luyn, W. N. Van Dijk-Wolthuis, et al., In vitro biocompatibility of biodegradable dextranbased hydrogels tested with human fibroblasts, Biomaterials, 2001, 22(11), 1197-1203.

112 E. M. Bachelder, T. T. Beaudette, K. E. Broaders, J. Dashe and J. M. Frechet, Acetal-derivatized dextran: an acidresponsive biodegradable material for therapeutic applications, J. Am. Chem. Soc., 2008, 130(32), 10494-10495.

113 J. Gilroy, M. I. Barnhart and J. S. Meyer, Treatment of acute stroke with dextran 40, J. Am. Med. Assoc., 1969, 210(2), 293298.

114 N. Lennard, J. Smith, J. Dumville, et al., Prevention of postoperative thrombotic stroke after carotid endarterectomy: the role of transcranial Doppler ultrasound, J. Vasc. Surg., 1997, 26(4), 579-584.

115 D. Gupta, C. H. Tator and M. S. Shoichet, Fast-gelling injectable blend of hyaluronan and methylcellulose for intrathecal, localized delivery to the injured spinal cord, Biomaterials, 2006, 27(11), 2370-2379.

116 B. D. Ratner, A. S. Hoffman, F. J. Schoen and J. E. Lemons, Biomaterials Science: An Introduction to Materials in Medicine, Elsevier Academic Press, Amsterdam, Boston, 2004.

117 W. S. Hu and D. I. Wang, Selection of microcarrier diameter for the cultivation of mammalian cells on microcarriers, Biotechnol. Bioeng., 1987, 30(4), 548-557.

118 E. K. Yim, S. W. Pang and K. W. Leong, Synthetic nanostructures inducing differentiation of human mesenchymal stem cells into neuronal lineage, Exp. Cell Res., 2007, 313(9), 1820-1829.

119 J. Yoo, M. Noh, H. Kim, N. L. Jeon, B. S. Kim and J. Kim, Nanogrooved substrate promotes direct lineage reprogramming of fibroblasts to functional induced dopaminergic neurons, Biomaterials, 2015, 45, 36-45.

120 S. E. Gratton, P. A. Ropp, P. D. Pohlhaus, et al., The effect of particle design on cellular internalization pathways, Proc. Natl. Acad. Sci. U. S. A., 2008, 105(33), 11613-11618.

121 M. Ni, W. H. Tong, D. Choudhury, N. A. Rahim, C. Iliescu and $\mathrm{H}$. Yu, Cell culture on MEMS platforms: a review, Int. J. Mol. Sci., 2009, 10(12), 5411-5441.

122 K. Fairman and B. S. Jacobson, Unique morphology of HeLa cell attachment, spreading and detachment from microcarrier beads covalently coated with a specific and non-specific substratum, Tissue Cell, 1983, 15(2), 167-180.

123 E. Walter, D. Dreher, M. Kok, et al., Hydrophilic poly(DLlactide-co-glycolide) microspheres for the delivery of DNA to human-derived macrophages and dendritic cells, $J$. Controlled Release, 2001, 76(1-2), 149-168.

124 L. T. Allen, E. J. Fox, I. Blute, et al., Interaction of soft condensed materials with living cells: phenotype/ transcriptome correlations for the hydrophobic effect, Proc. Natl. Acad. Sci. U. S. A., 2003, 100(11), 6331-6336.

125 S. Zhuo, M. Ni, L. Bi, L. Xia, J. Fan and H. Chan, Stem CellBiomaterial Interactions for Tissue Engineering, Stem Cells Int., 2015, 126710.

126 G. Klein, M. Langegger, R. Timpl and P. Ekblom, Role of laminin A chain in the development of epithelial cell polarity, Cell, 1988, 55(2), 331-341.

127 K. Yang, J. S. Lee, J. Kim, et al., Polydopamine-mediated surface modification of scaffold materials for human neural stem cell engineering, Biomaterials, 2012, 33(29), 6952-6964.

128 D. S. Benoit, M. P. Schwartz, A. R. Durney and K. S. Anseth, Small functional groups for controlled differentiation of hydrogel-encapsulated human mesenchymal stem cells, Nat. Mater., 2008, 7(10), 816-823.

129 A. J. Engler, S. Sen, H. L. Sweeney and D. E. Discher, Matrix elasticity directs stem cell lineage specification, Cell, 2006, 126(4), 677-689.

130 S. Ilkhanizadeh, A. I. Teixeira and O. Hermanson, Inkjet printing of macromolecules on hydrogels to steer neural stem cell differentiation, Biomaterials, 2007, 28(27), 39363943.

131 K. Jin, X. Mao, L. Xie, et al., Transplantation of human neural precursor cells in Matrigel scaffolding improves outcome from focal cerebral ischemia after delayed postischemic treatment in rats, J. Cereb. Blood Flow Metab., 2010, 30(3), 534-544.

132 V. M. Tatard, M. C. Venier-Julienne, P. Saulnier, et al., Pharmacologically active microcarriers: a tool for cell therapy, Biomaterials, 2005, 26(17), 3727-3737.

133 N. Daviaud, E. Garbayo, L. Sindji, A. Martinez-Serrano, P. C. Schiller and C. N. Montero-Menei, Survival, differentiation, and neuroprotective mechanisms of human stem cells complexed with neurotrophin-3releasing pharmacologically active microcarriers in an ex vivo model of Parkinson's disease, Stem Cells Transl. Med., 2015, 4(6), 670-684.

134 C. Bouffi, O. Thomas, C. Bony, et al., The role of pharmacologically active microcarriers releasing TGFbeta3 in cartilage formation in vivo by mesenchymal stem cells, Biomaterials, 2010, 31(25), 6485-6493. 